\title{
Epidemiology and clinical characteristics of severe non-polio enteroviruses infections: the pediatric experience of the National Institute for Infectious Diseases "Prof. Dr. Matei Balş", a 5 year survey
}

\author{
Monica Luminos ${ }^{1,2^{*}}$, Gheorghiță Jugulete ${ }^{1,2}$, Angelica Vişan ${ }^{1,2}$, Magda Vasile ${ }^{1}$, Anuța Bilaşco ${ }^{1}$, Anca Drăgănescu' , \\ Sabina Șchiopu ${ }^{1}$, Cornelia Dogaru', Mădălina Maria Merişescu ${ }^{1,2}$, Cristina Negulescu' ${ }^{1}$, Osman Endis $^{1}$
}

From The 9th Edition of the Scientific Days of the National Institute for Infectious Diseases Prof Dr Matei Bals Bucharest, Romania. 23-25 October 2013

\section{Background}

Currently, an increase in the enteroviruses associated diseases is reported in many countries of the world. Understanding the trend of severe non-polio enteroviruses infections, in the pediatric population, becomes an important public health issue.

\section{Methods}

We analyzed the clinical manifestations of patients aged 1 month to 14 years, with serology-confirmed non-polio enteroviral infections, who were hospitalized for at least 4 days in the National Institute for Infectious Diseases "Prof. Dr. Matei Balş", between January 2009 and September 2013.

\section{Results}

There were 63 laboratory-confirmed enteroviruses severe infections during this period. In our cohort $67 \%$ of the patients were males and the median age was $4.2 \pm 2.2$ years of age. The average days from onset to deterioration were 4.3 days (range 1-10 days). Interestingly, more than half did not have oral ulcers, and/or characteristic skin rashes, thus making earlier diagnosis more difficult. The vast majority ( $92 \%$ of the patients) associated gastrointestinal manifestations.

A total of 23 patients were diagnosed with severe nonpolio enterovirus aseptic meningitis, with the highest prevalence of the cases in the summer of 2012, when
18 children developed acute flaccid paralysis, 13 manifested non-specific febrile skin rashes and 9 presented with viral hepatitis.

The average time of hospitalization was 6.4 days (range 4-31 days) and all patients required interdisciplinary consults, 12 required kineto-therapy after discharge but neither one of our patients had a fatal outcome.

\section{Conclusion}

Even in the absence of typical oral ulcers, skin rashes and/ or gastrointestinal symptoms, clinicians should be alert about any flaccid paralysis episode and other neurological signs.

\section{Authors' details \\ ${ }^{1}$ National Institute for Infectious Diseases "Prof. Dr. Matei Balş", Bucharest, Romania. ${ }^{2}$ Carol Davila University of Medicine and Pharmacy, Bucharest,} Romania.

Published: 16 December 2013

doi:10.1186/1471-2334-13-S1-P102

Cite this article as: Luminos et al.: Epidemiology and clinical characteristics of severe non-polio enteroviruses infections: the pediatric experience of the National Institute for Infectious Diseases "Prof. Dr. Matei Balş", a 5 year survey. BMC Infectious Diseases 2013 13(Suppl 1):P102.

\footnotetext{
* Correspondence: drluminos@mateibals.ro

${ }^{1}$ National Institute for Infectious Diseases "Prof. Dr. Matei Balş", Bucharest, Romania

Full list of author information is available at the end of the article
} 